\title{
Engineering and application of synthetic nar promoter for fine-tuning the expression of metabolic pathway genes in Escherichia coli
}

Hee Jin Hwang' ${ }^{1}$ Sang Yup Lee ${ }^{2}$ and Pyung Cheon Lee ${ }^{1^{*}}$

\begin{abstract}
Background: Promoters regulate the expression of metabolic pathway genes to control the flux of metabolism. Therefore, fine-tuning of metabolic pathway gene expression requires an applicable promoter system. In this study, a dissolved oxygen-dependent nar promoter was engineered for fine-tuning the expression levels of biosynthetic pathway enzymes in Escherichia coli. To demonstrate the feasibility of using the synthetic nar promoters in production of biochemicals in E. coli, the D-lactate pathway consisting of one enzyme and the 2,3-butanediol (BDO) pathway consisting of three enzymes were investigated.

Results: The spacer sequence of 15 bp between the -35 and -10 elements of the upstream region of the wildtype nar promoter was randomized, fused to the GFP gene, transduced into E. coli, and screened by flow cytometry. The sorted synthetic nar promoters were divided into three groups according to fluorescence intensity levels: strong, intermediate, and weak. The selected three representative nar promoters of strong, intermediate, and weak intensities were used to control the expression level of the D-lactate and 2,3-BDO biosynthetic pathway enzymes in E. coli. When the IdhD gene encoding D-lactate dehydrogenase was expressed under the control of the strong synthetic nar promoter in fed-batch cultures of E. coli, the D-lactate titers were $105.6 \mathrm{~g} / \mathrm{L}, 34 \%$ higher than those using the wild-type promoter (79.0 g/L). When the three 2,3-BDO pathway genes ( $i / v B N$, aldB, and bdh 1 ) were expressed under the control of combinational synthetic nar promoters (strong-weak-strong) in fed-batch cultures of E. coli, the titers of 2,3-BDO were $88.0 \mathrm{~g} / \mathrm{L}, 72 \%$ higher than those using the wild-type promoter $(51.1 \mathrm{~g} / \mathrm{L})$.

Conclusions: The synthetic nar promoters, which were engineered to have strong, intermediate, and weak intensities, were successfully applied to metabolic engineering of D-lactate and 2,3-BDO pathways in E. coli. By controlling expression levels of D-lactate and 2,3-BDO pathway enzymes using the synthetic nar promoters, the production of D-lactate and 2,3-BDO was increased over that using the wild-type promoter by 34 and $72 \%$, respectively. Thus, this synthetic promoter module system will support the improved production of biochemicals and biofuels through finetuning of gene expression levels.
\end{abstract}

Keywords: nar promoter, Oxygen-dependent promoter, Lactate, 2,3-Butanediol, Promoter engineering

\footnotetext{
*Correspondence: pclee@ajou.ac.kr

1 Department of Molecular Science and Technology, Ajou University,

Woncheon-dong, Yeongtong-gu, Suwon 16944, South Korea

Full list of author information is available at the end of the article
} 


\section{Background}

Synthetic biology aims to develop desired biological system through the rational design of synthetic parts/ modules, including promoters, RNAs, and scaffolds [1-4]. Gene expression can be controlled utilizing several factors, such as promoters, transcription factors, and plasmid copy numbers [5, 6]. Among them, promoter engineering has been proposed as one of the most efficient ways of fine-tuning transcriptional control in Escherichia coli, Corynebacterium glutamicum, Bacillus subtilis, and yeasts [3-10]. For example, the E. coli strain with engineered L-phenylalanine-responsive promoter could produce fourfold higher titer of phenylalanine than wild-type promoter [11], and the engineered tac promoter library could decrease leakage of antibody fragment expression in E. coli [12].

Recently, a dissolved oxygen (DO)-dependent nar promoter was successfully applied to express the D-lactate, 2,3-butanediol (BDO), and 1,3-propanediol (PDO) pathway enzymes in E. coli [13]. However, when a multienzyme biosynthetic pathway was reconstructed in heterologous host cells, individual expression of each pathway enzyme needed to be finely controlled; assembly or organization of multienzyme systems could significantly influence metabolic channeling, and thus suboptimal assembly or organization would cause accumulation of unwanted metabolic intermediates in multistep enzyme reactions [14, 15]. Even single-enzyme metabolic pathways also should be considered for finetuning of expression, because expression level frequently affects end-product formation due to inclusion body formation [16].

Compared to other commonly used strong promoters such as lac or $\operatorname{araBAD}$, the intensity of the wild-type nar promoter is relatively weak [13]; therefore, engineering of the wild-type nar promoter was required for fine control of target pathway gene expression. In this study, in order to generate synthetic nar promoters of diverse strengths, a synthetic nar promoter library was constructed by randomization of the spacer region sequence ( $15 \mathrm{bp}$ ) located between the consensus sequence -35 and -10 elements of the wild-type nar promoter (Fig. 1a). Characterization of the selected three synthetic promoters showing weak, intermediate, and strong intensities was performed through transcriptional expression level and GFP fluorescence intensity assays. Then, the selected three synthetic promoters were applied to the expression of the $\mathrm{D}$-lactate pathway consisting of one gene and the 2,3-BDO biosynthesis pathway consisting of three genes (Fig. 1b). We demonstrated that the production of D-lactate and 2,3-BDO was improved by tuning the expression of their pathway genes under three different strengths of the synthetic nar promoters in E. coli.

\section{Results}

Construction, screening, and strength analysis of a synthetic nar promoter library of diverse strengths

The 15-bp spacer region sequence between the -35 and -10 elements of the wild-type nar promoter was randomized using degenerated primers to construct a synthetic nar promoter library of diverse strengths. The randomized synthetic promoters were fused by PCR to a DNA fragment consisting of a Shine-Dalgarno sequence, spacer, and $\mathrm{His}_{6}$-tagged GFPm as a reporter protein for screening based on the fluorescence intensity of the expressed GFPm (Fig. 1a). The randomized promoter fragments were then ligated with a pSTVM plasmid (Table 1) and then transduced into $E$. coli TOP10 cells.

The library $\left(4.59 \times 10^{10}\right.$ cell size $)$, showing diverse fluorescence intensities, was sorted into three groups (low, intermediate, and high strength) by FACS in three rounds of sorting. The 1 st round sorting was carried out with two collections of the upper and lower $1 \%$ of fluorescence intensity signals. The collected cells were grown on $\mathrm{LB}+\mathrm{cm}$ agar plates and submitted to 2 nd round sorting. After repeating this for 3rd round sorting, clones showing high (a red line in Fig. 2a), immediate (a blue line), and low (a yellow line) fluorescence intensities were collected and further analyzed.

After reconfirmation of the GFP fluorescence intensities of 300 randomly selected clones from the 3 groups (100 clones from each group), fluorescences of 68 distinguishable clones were measured and compared based on relative fluorescence units (RFU)/OD ${ }_{600}$ (Fig. 2b). Based on the normalized values of RFU/OD 600 , synthetic nar promoters were grouped into strong ( $\left.>5000 \mathrm{RFU} / \mathrm{OD}_{600}\right)$, intermediate (1000-2000 RFU/OD 600$)$, and weak $(<1000$ RFU/OD ${ }_{600}$ ) groups. From each group, a representative synthetic nar promoter of strong (S3-2-64), intermediate (W2U-30), and weak (W2L-29) fluorescence was chosen for further analysis. The strength of the strong synthetic nar promoter (S3-2-64) and the intermediate nar promoter (W2U-30) were 19.8 and 6.2 times higher than that of the wild-type nar promoter, respectively, while the strength of the weak nar promoter (W2L-29) was 1.8 times weaker than that of the wild-type nar promoter based on values of RFU/OD 600 .

\section{Characterization of the three representative synthetic nar promoters}

The three representative nar synthetic promoters were then characterized in detail by analyzing levels of transcription, protein expression, and fluorescence of GFPm (Fig. 3a). In transcriptional analysis, qRT-PCR was carried out with a cysG gene encoding siroheme synthase [17] as a reference in order to evaluate $\Delta \Delta C_{t}$ values of GFPm expression under the control of the wild-type, 


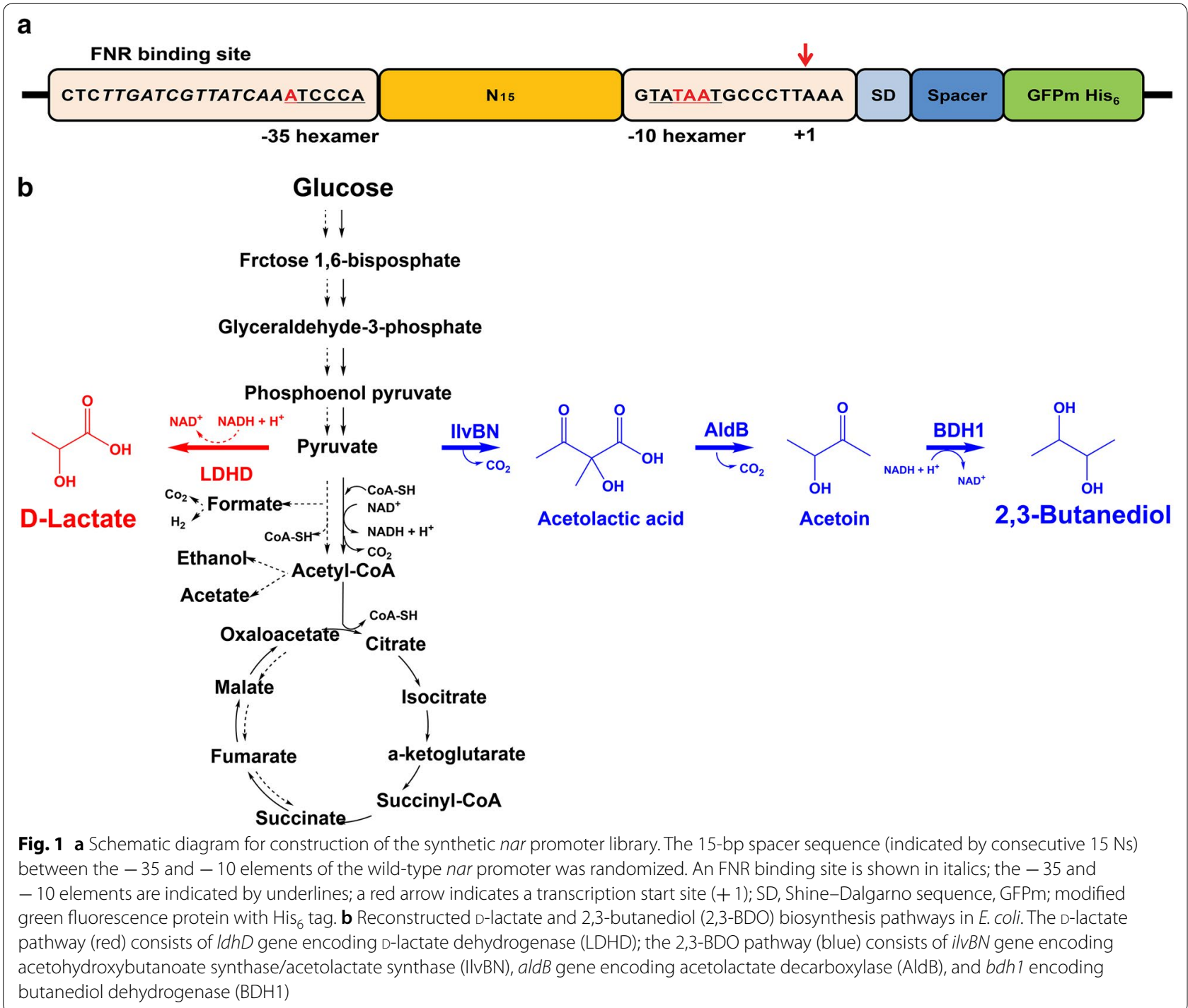

strong, intermediate, and weak nar promoters. The $\Delta \Delta C_{\mathrm{t}}$ values of the strong, intermediate, and weak nar promoters were $29.4 \pm 5.6,8.4 \pm 1.0$, and $2.3 \pm 0.4$, respectively. Unexpectedly, the $\Delta \Delta C_{\mathrm{t}}$ value of the weak nar promoter was positive, indicating a higher transcription level than that of the wild-type nar promoter. Next, protein expression levels were examined by western blotting with endogenous GAPDH as a reference. The protein expression levels of GFPm under the control of the strong, intermediate, and weak nar promoters were approximately 19.7, 7.11, and 0.37 times higher than that of the wild-type nar promoter. Notably, the protein expression levels of the strong and intermediate nar promoters were 4.6 and 1.7 times higher, respectively, than that of the constitutive lac promoter. Finally, the fluorescence intensities of GFPm under the control of the strong, intermediate, and weak nar promoters were $10,017 \pm 915$, $2305 \pm 360$, and $218 \pm 18 \mathrm{RFU} / \mathrm{OD}_{600}$, respectively.

The randomly mutated spacer regions of the nine nar promoters including three representatives were sequenced and compared. One distinguishable difference between the synthetic and wild-type nar promoters was a GC content in the space sequences: lower GC contents were observed in all synthetic promoters (strong: 33.3\%; intermediate: $40 \%$; weak: $33.3 \%$ ) than in the wild-type nar promoter (60\%) (Fig. 3b). A conserved sequence (GTN[A/G]N) located between the -24 and -20 positions was observed in seven clones representing intermediate and weak promoters, but not in that of the strong promoter. It has been shown that strong promoters tend to have AT-rich spacers, which help flexibility and bendability of DNA structures [18]. This is true for the strong 
Table 1 Bacterial strains and plasmids used in this study

\begin{tabular}{|c|c|c|}
\hline Strains and plasmids & Relevant properties & Source or reference \\
\hline \multicolumn{3}{|l|}{ Strains } \\
\hline E. coliTOP10 & $\begin{array}{l}\text { F-mcrA } \Delta(\text { mrr-hsdRMS-mcrBC) } \varphi 80 l a c Z \Delta M 15 \Delta l a c X 74 \text { nupG recA1 araD139 } \Delta \text { (ara-leu)7697 galE15 } \\
\text { galK16 rpsL(Str }{ }^{R} \text { ) endA1 }\end{array}$ & Invitrogen \\
\hline W023 & W $\triangle l d h A \triangle p f l B \triangle a d h E \Delta l p d A: K . p . I p d E 354 K \Delta m d h \triangle \operatorname{arcA} g \mid t A R 164 L$ & {$[13,21]$} \\
\hline Lactobacillus citreum & Source for $I d h D$ & КСТС 3721 \\
\hline Lactococcus lactis subsp. lactis & Source for aldB & КСТС3899 \\
\hline Saccharomyces cerevisiae S288c & Source for bdh1 & ATCC 204508 \\
\hline \multicolumn{3}{|l|}{ Plasmids } \\
\hline pUCM & Cloning vector modified from pUC19; constitutive lac promoter, $A p^{R}$ & {$[23]$} \\
\hline pUCM-gfpm & Constitutive expressed gfpm gene with lac promoter & This study \\
\hline pUCN & Cloning/expression vector having inducible wild-type nar promoter, $\mathrm{Amp}^{R}$ & [13] \\
\hline pUCN-gfpm & Inducible expressed gfpm gene with nar promoter & This study \\
\hline pQE-gfpm & Inducible expressed gfpm gene with T5 promoter & {$[22]$} \\
\hline $\mathrm{pUCNr}$ & Cloning/expression vector having rop gene and wild-type nar promoter, $\mathrm{Amp}^{R}$ & This study \\
\hline pUCNrS & Cloning/expression vector having rop gene and strong nar promoter (S3-2-64), Amp ${ }^{R}$ & This study \\
\hline pUCNrl & Cloning/expression vector having rop gene and intermediate nar promoter (W2U-30), Amp ${ }^{R}$ & This study \\
\hline pUCNrW & Cloning/expression vector having rop gene and weak nar promoter (W2L-29), Amp ${ }^{R}$ & This study \\
\hline $\mathrm{NrSL}$ & Inducible expressed IdhD gene from L. citreum on pUCNrS & This study \\
\hline $\mathrm{NrIL}$ & Inducible expressed IdhD gene from L. citreum on pUCNrl & This study \\
\hline NrWL & Inducible expressed IdhD gene from L. citreum on pUCNrW & This study \\
\hline $\mathrm{NrSi}$ & Inducible expressed ilvBN gene from E. coli on pUCNrS & This study \\
\hline Nrli & Inducible expressed ilvBN gene from E. coli on pUCNrl & This study \\
\hline NrWi & Inducible expressed ilvBN gene from E. coli on pUCNrW & This study \\
\hline $\mathrm{NrSa}$ & Inducible expressed aldB gene from L. lactis with strong nar promoter & This study \\
\hline Nrla & Inducible expressed aldB gene from L. lactis with intermediate nar promoter & This study \\
\hline NrWa & Inducible expressed aldB gene from L. lactis with weak nar promoter & This study \\
\hline $\mathrm{NrSb}$ & Inducible expressed bdh1 gene from S. cerevisiae with strong nar promoter & This study \\
\hline Nrlb & Inducible expressed bdh1 gene from S. cerevisiae with intermediate nar promoter & This study \\
\hline $\mathrm{NrWb}$ & Inducible expressed bdh1 gene from S. cerevisiae with weak nar promoter & This study \\
\hline pSTVM2 & Cloning/expression vector removing lac promoter, $\mathrm{Cm}^{\mathrm{R}}$ & [13] \\
\hline pSTVM2-SNPL-gfpm & gfpm expression vector with synthetic nar promoter library & This study \\
\hline pSTVM-gfpm & Constitutive expressed gfpm gene with constitutive lac promoter on pSTVM2 & This study \\
\hline $\mathrm{Ni}$ & Inducible expressed ilvBN gene with wild-type nar promoter on pSTVM2 & [13] \\
\hline $\mathrm{Si}$ & Inducible expressed ilvBN gene with strong nar promoter on pSTVM2 & This study \\
\hline li & Inducible expressed ilVBN gene with intermediate nar promoter on pSTVM2 & This study \\
\hline Wi & Inducible expressed ilvBN gene with weak nar promoter on pSTVM2 & This study \\
\hline $\mathrm{NiNa}$ & $\begin{array}{l}\text { Individually inducible expressed ilVBN and aldB genes with wild-type nar promoter on } \\
\text { PSTVM2 }\end{array}$ & [13] \\
\hline SiSa & Individually inducible expressed ilVBN and aldB genes with strong nar promoter on pSTVM2 & This study \\
\hline lila & $\begin{array}{l}\text { Individually inducible expressed ilvBN and aldB genes with intermediate nar promoter on } \\
\text { pSTVM2 }\end{array}$ & This study \\
\hline SiWa & $\begin{array}{l}\text { Individually inducible expressed ilvBN with strong promoter and aldB with weak nar promoter } \\
\text { on PSTVM2 }\end{array}$ & This study \\
\hline NNN & Inducible expressed ilvBN, aldB, and bdh1 genes under each nar promoter on pSTVM2 & [13] \\
\hline SWS & $\begin{array}{l}\text { Individually inducible expressed ilvBN with strong promoter, aldB with weak promoter, and } \\
b d h 1 \text { with strong promoter }\end{array}$ & This study \\
\hline
\end{tabular}



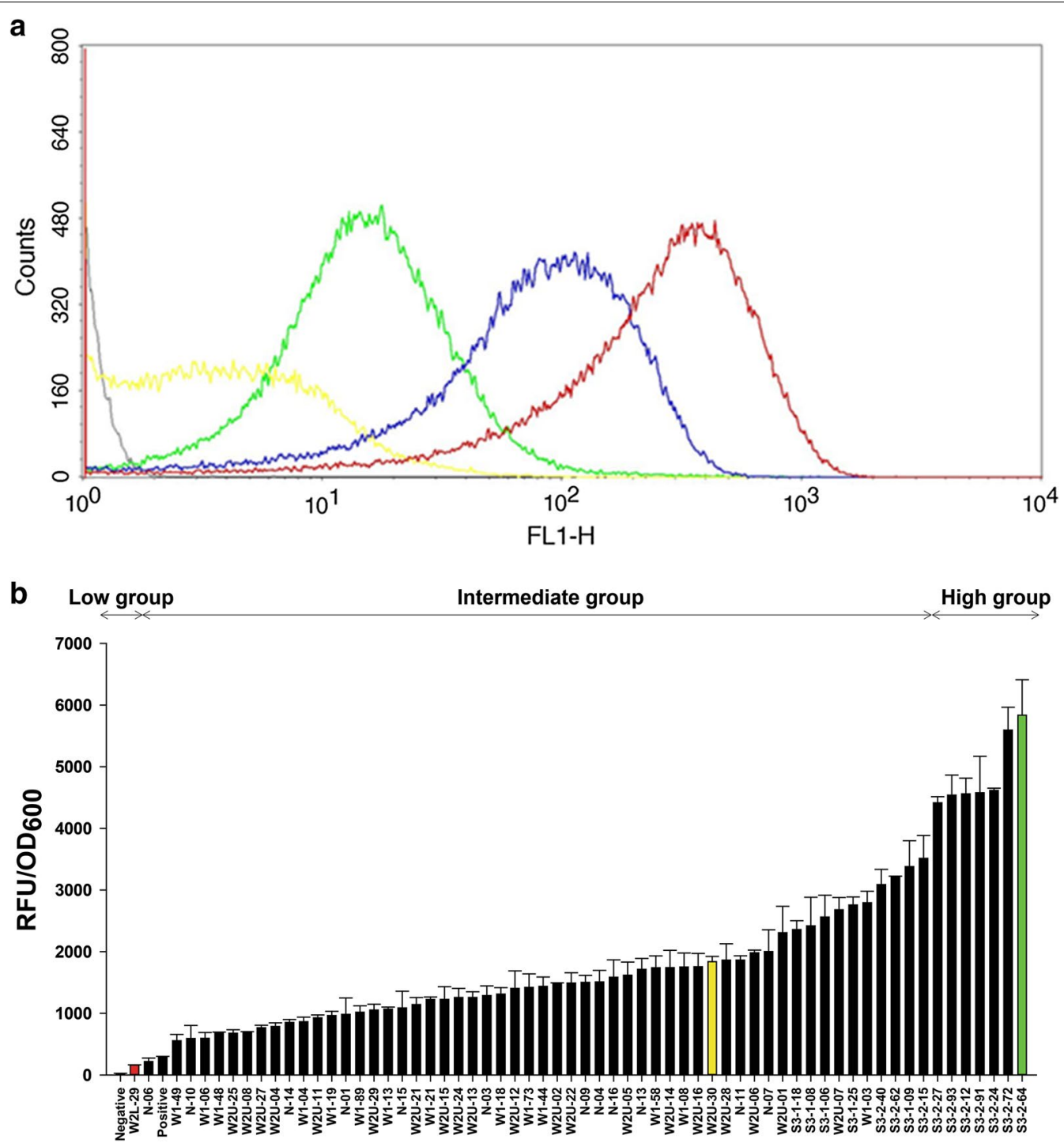

Fig. 2 a FACS analysis of E. coli cells containing the synthetic nar promoter library. Histograms of sorted cell libraries and control cells are represented by colored lines. Black: negative control (empty vector), yellow: sorted cells showing weak intensities, green: positive control (a wild-type nar promoter), blue: sorted cells showing intermediate intensities, and red: sorted cells showing strong intensities. $\mathbf{b}$ Promoter strength analysis of the synthetic nar promoters. GFP fluorescence intensities of the collected 68 clones having synthetic nar promoters were measured by spectrofluorometer and normalized as relative fluorescence unit (RFU)/ $\mathrm{OD}_{600}$. Representative strong (S3-2-64), intermediate (W2U-30), and weak (W2L-29) nar promoters are represented by green, yellow, and red bars, respectively

nar promoter because it has AT-rich spacers and lacks the conserved sequence present in the weaker promoters.

\section{Comparison of D-lactate production with synthetic nar promoters of different strengths}

In order to investigate the effect of nar promoter strength on metabolic pathway flux in E. coli, a D-lactate pathway consisting of one D-lactate dehydrogenase was first chosen. The $l d h D$ gene encoding D-lactate dehydrogenase from L. citreum was cloned into pUCNrS, pUCNrI,
pUCNrW, and pUCN (Table 1), to be expressed under the control of the four nar promoters: strong, intermediate, weak, and wild-type. The four $l d h D$ gene-expression plasmids (NrSL, strong; NrIL, intermediate; NrWL, weak; NrL, wild-type) were transduced into $E$. coli, and then the four recombinant $E$. coli strains were microaerobically grown in flasks containing $20 \mathrm{~g} / \mathrm{L}$ glucose as a carbon source [13]. After a 20 -h cultivation, D-lactate titers were measured to be $18.6 \pm 0.6$ in E. coli having $\mathrm{NrL}, 18.7 \pm 0.4$ in NrSL, $18.5 \pm 0.4$ in NrIL, and $18.3 \pm 0.1$ 


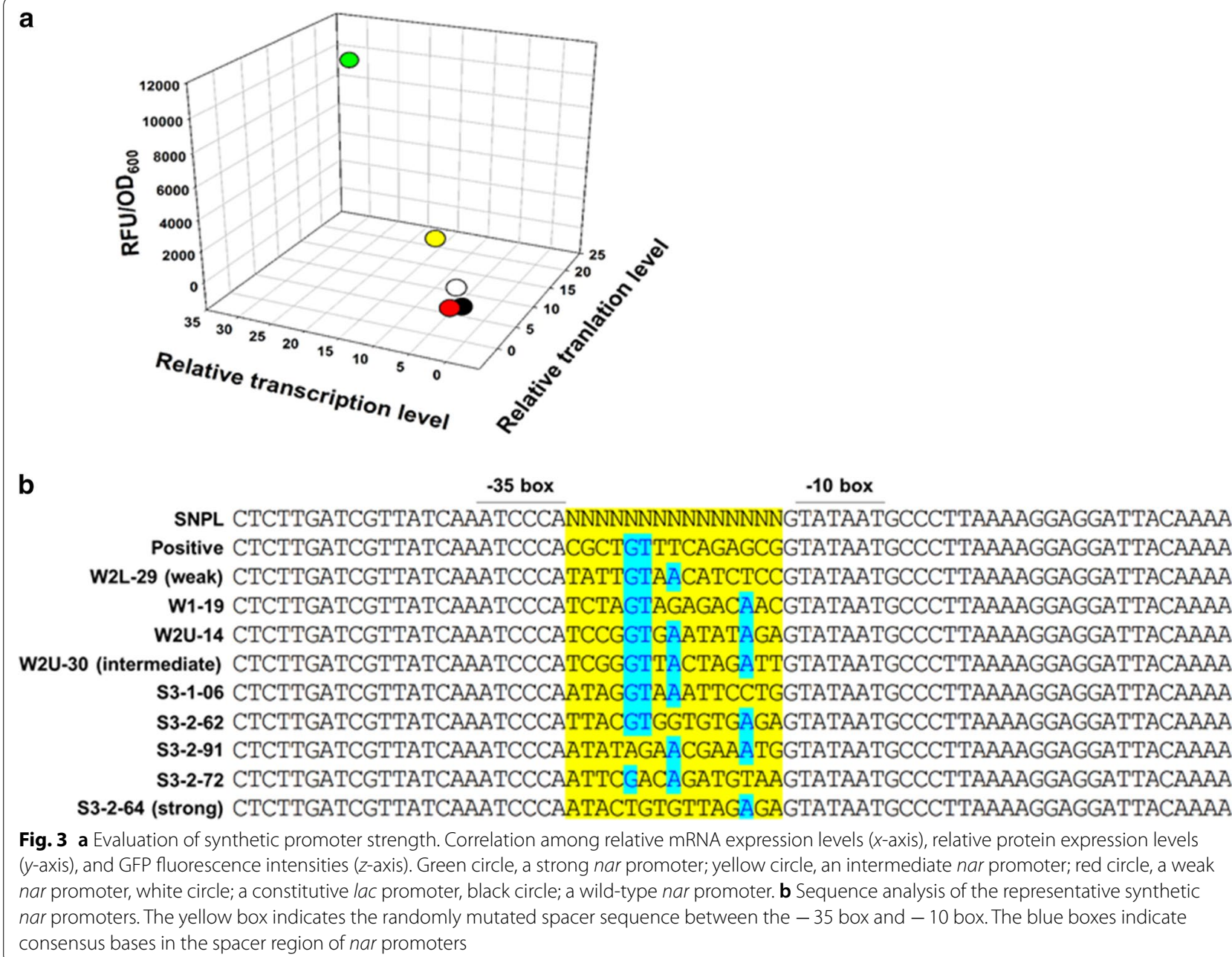

in NrWL (Fig. 4a). Because D-lactate titers were similar in all four E. coli strains, two E. coli strains having $\mathrm{NrL}$ and NrSL were chosen and fed-batch fermentation with glucose as a carbon source was carried out to investigate the effect of the strength of nar promoters (strong vs. wild-type) on D-lactate production. When the DOcontrolled fed-batch fermentation of $E$. coli strain having NrSL was carried out as described in our previous study [13], $105.6 \mathrm{~g} / \mathrm{L}$ of D-lactate was obtained after a 23-h cultivation. The D-lactate yield and productivity were $0.71 \mathrm{~g} / \mathrm{g}$-glucose and $4.59 \mathrm{~g} / \mathrm{L} / \mathrm{h}$, respectively (Fig. $4 \mathrm{~b}$ ). In comparison, the $E$. coli strain with NrL produced $79.0 \mathrm{~g} / \mathrm{L}$ of D-lactate with D-lactate yield of $0.67 \mathrm{~g} / \mathrm{g}$-glucose and productivity of $3.47 \mathrm{~g} / \mathrm{L} / \mathrm{h}$ (Fig. 4c). This result supports that higher expression of D-lactate dehydrogenase under the control of the synthetic strong nar promoter directed more metabolic flux into D-lactate biosynthesis in E. coli. Consequently, controlling D-lactate dehydrogenase with the strong nar promoter enhanced D-lactate titers by
$34 \%$ compared to those of the wild-type nar promoter $(105.6 \mathrm{~g} / \mathrm{L}$ vs. $79.0 \mathrm{~g} / \mathrm{L})$.

\section{Comparison of acetoin and 2,3-BDO production with the synthetic nar promoters of different strengths}

As a second demonstration of the feasibility of using the synthetic nar promoters in production of biochemicals in $E$. coli, acetoin, consisting of two enzymes, and the 2,3-butanediol (2,3-BDO) pathway, consisting of three enzymes, were investigated using the three representative nar promoters. First, the production of acetoin, a 2,3BDO pathway intermediate (Fig. 1b), was investigated. In order to reconstruct the heterologous acetoin pathway in $E$. coli, two acetoin pathway genes, $i l v B N$ from $E$. coli and aldB from L. lactis, were modified to be expressed under the control of the three synthetic nar promoters by cloning each gene into pUCNrS, pUCNrI, and pUC$\mathrm{NrW}$. Next, the resulting 6 expression modules $(\mathrm{Si}, \mathrm{Ii}, \mathrm{Wi}$, $\mathrm{Sa}$, Ia, and Wa, where $\mathrm{S}$ stands for strong promoter, I for intermediate promoter, $\mathrm{W}$ for weak promoter, i for $i l v B N$, 

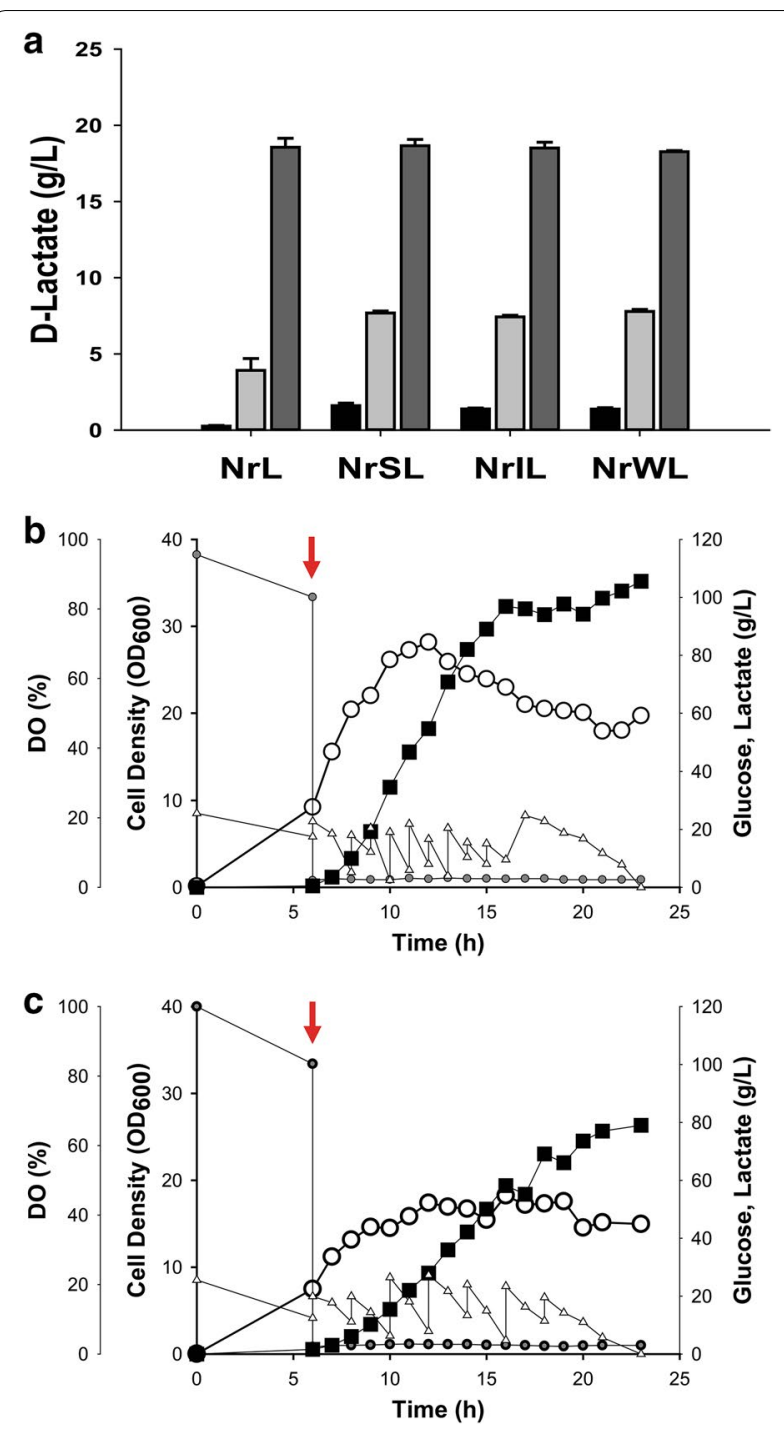

Fig. 4 a Comparison of D-lactate production by each promoter in flask-scale fermentations. The black, light gray, and dark gray indicate production of D-lactate after 4, 8, and $20 \mathrm{~h}$, respectively. The error bars show the standard deviations from triplicate experiments. Production of D-lactate under control of strong synthetic nar promoter (b) and the wild-type nar promoter (c) in fed-batch fermentations. Black square, D-lactate; hollow circle, cell growth; hollow triangle, glucose; solid gray circle, DO level; red arrows, the time of DO downshift (induction)

and a for aldB) were combined to produce 9 expression vectors (Fig. 5a).

When 10 expression modules including a positive control module $\mathrm{Ni}-\mathrm{Na}$ ( $i l v B N$ and $a l d B$ expressed with a wild-type nar promoter) were expressed in flask cultures of $E$. coli, the expression of the $i l v B N$ gene with strong and intermediate synthetic nar promoters (Si and Ii) produced an average of $6.30 \mathrm{~g} / \mathrm{L}$ of acetoin regardless of promoter strength for expressing the aldB gene. However, the expression of $i l v B N$ with the weak nar promoter (Wi) produced the lowest acetoin production $(3.4 \pm 0.3 \mathrm{~g} / \mathrm{L})$ when the $a l d B$ gene was coexpressed with the weak nar promoter (Wa) (Fig. 5b). Unexpectedly, when a heterologous acetoin pathway was reconstructed in $E$. coli, the end-product acetoin was to a limited degree transformed into 2,3-BDO by unknown factors [19]. Therefore, when the total summed amount of acetoin and 2,3-BDO produced by combination of the 10 modules was taken into consideration, $\mathrm{Si}-\mathrm{Wa}$ and $\mathrm{Ii}-\mathrm{Ia}$ combinations exhibited better production than the others $(8.4 \pm 0.5$ and $8.1 \pm 0.6 \mathrm{~g} / \mathrm{L}$, respectively).

Next, the two selected acetoin-producing combination modules, $\mathrm{Si}-\mathrm{Wa}$ and $\mathrm{Ii}-\mathrm{Ia}$, were assembled as independent expression modules into one plasmid pSTVM2, resulting in SiWa and Iila (Table 1). In order to reconstruct a 2,3-BDO pathway on the two-plasmid system, SiWa and Iila were coexpressed with $b d h 1$ from $S$. cerevisiae under the control of the 3 synthetic nar promoters (6 expression combinations: Si, Ii, Wi, Sa, Ia, Wa, Sb, $\mathrm{Ib}$, and $\mathrm{Wb}$, where $\mathrm{S}$ stands for strong, I for intermediate, W for weak, i for $i l v B N$, a for aldB, and b for $b d h 1$; Fig. 6a) in flask cultures. Among seven complementations including a wild-type nar promoter module (NiNa$\mathrm{Nb})$, the SiWa-Sb combination module produced the highest titer of 2,3-BDO $(9.7 \pm 0.2 \mathrm{~g} / \mathrm{L})$, followed by the SiWa-Wb module (Fig. 6b). This best combination modules along with the wild-type nar promoter module as a positive control were then assembled as an independent expression module into one plasmid pSTVM2, resulting in SWS and NNN. After fed-batch cultivation of E. coli expressing NNN and SWS, the SWS module produced $88.0 \mathrm{~g} / \mathrm{L}$ of 2,3-BDO (Fig. 6c), while the NNN module produced $51.1 \mathrm{~g} / \mathrm{L}$ of 2,3-BDO (Fig. 6d). This fine-tuning of each 2,3-BDO pathway enzyme expression enhanced the $2,3-\mathrm{BDO}$ titer by $72 \%$. Even though conversion yields (g/g-glucose) were similar [0.33 (NNN) vs. 0.35 (SWS)], volumetric productivity of 2,3-BDO obtained by expressing SWS was $1.87 \mathrm{~g} / \mathrm{L} / \mathrm{h}$, which was $75 \%$ higher than the $1.07 \mathrm{~g} / \mathrm{L} / \mathrm{h}$ obtained by expressing NNN.

\section{Discussion}

A promoter is one cellular strategy for controlling the flux of metabolism by regulating the expression of metabolic pathway genes. Therefore, fine-tuning of metabolic pathway gene expression requires an applicable promoter system. In our previous study, a DO-dependent nar promoter was successfully applied to control expressions of biochemical biosynthetic pathway enzymes in E. coli [13]. However, more fine-tuning of expressions of biochemical biosynthetic pathway enzymes is necessary to enhance yield and titer of a target biochemical or 

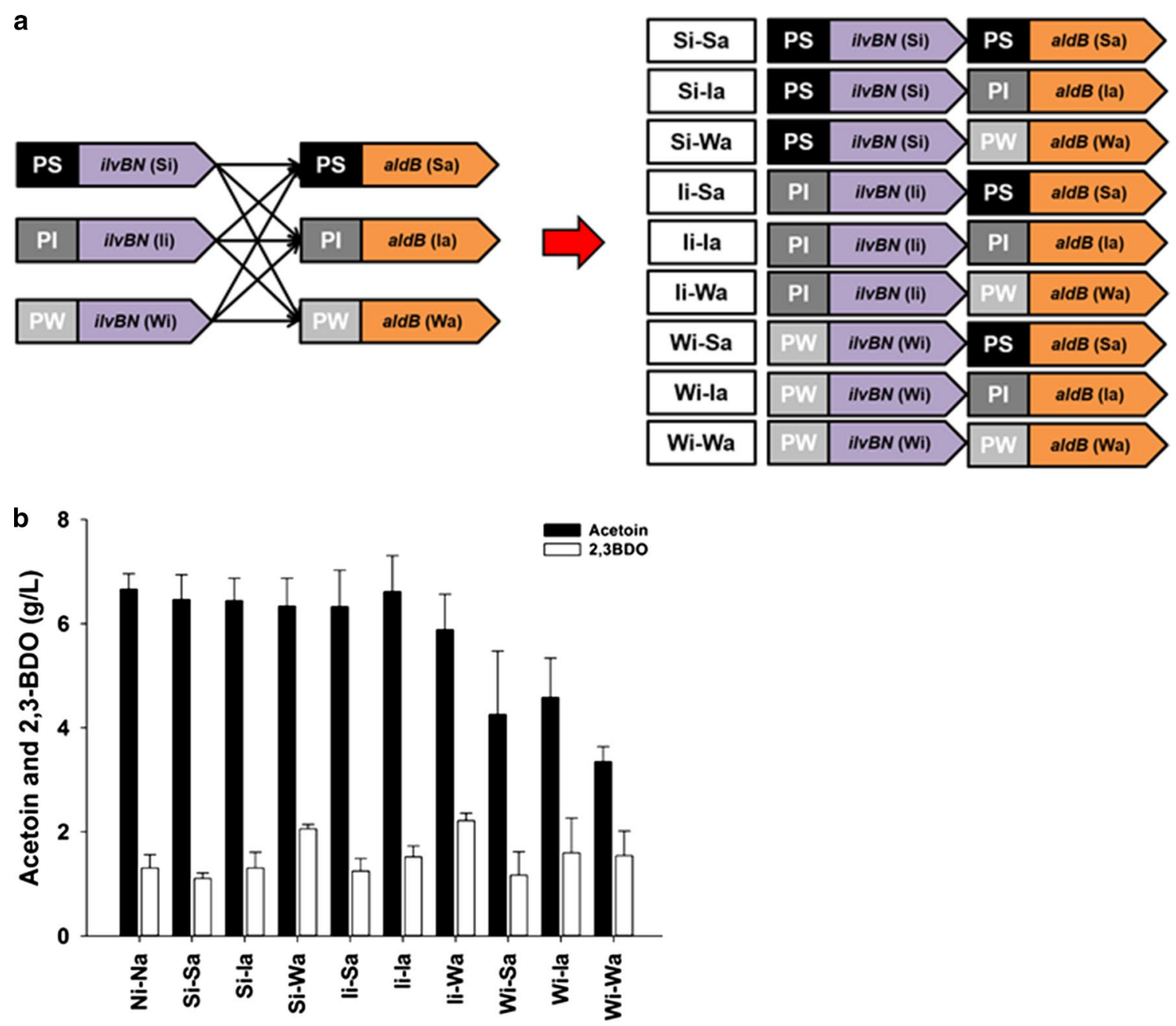

Fig. 5 Comparison of acetoin production with the expression of $i / v B N$ and aldB under control of the 3 synthetic nar promoters in flask cultures. a Combination of expression modules of ilvBN and aldB genes. Modules are Si, li, Wi, Sa, la, and Wa; S stands for strong promoter, I for intermediate promoter, $\mathrm{W}$ for weak promoter, i for ilvBN, and a for aldB. $\mathbf{b}$ Titer of acetoin obtained by expressing 10 modules in flask cultures. The positive control $\mathrm{Ni}-\mathrm{Na}$ is a module expressing the ilvBN and aldB genes with a wild-type nar promoter. Black and white bars indicate acetoin and 2,3-BDO, respectively

biofuel by metabolic flux channeling. Therefore, in this study, 3 synthetic nar promoters showing weak (W2L29), intermediate (W2U-30), and strong (S3-2-64) intensities were constructed by randomization of the spacer region sequence ( $15 \mathrm{bp}$ ) located between the consensus sequence -35 and -10 elements of the wild-type nar promoter (Fig. 1a). Analyses of transcription by qRTPCR, protein expression by western blotting, and fluorescence by a GFPm reporter protein assay were in a good correlation with the apparent strengths of the 3 synthetic nar promoters (Fig. 3a). Sequence analysis showed that these synthetic nar promoters have relatively higher AT contents than the wild-type nar promoter and higher variations at the $-24,-20$, and -14 sequence sites
(Fig. 3b). The observed features of the synthetic nar promoters are well supported by other research reporting that the AT-rich sequences of the spacer region affected the strength of the promoter through structural changes $[18,20]$.

To evaluate the general use of synthetic promoters in biochemical production, the 3 synthetic nar promoters were used to express D-lactate and 2,3-BDO pathway enzymes. Among the 3 synthetic nar promoters, the expression of the $l d h D$ gene under the control of the strong synthetic nar promoter on a low-copy plasmid produced the highest amount $(105.6 \mathrm{~g} / \mathrm{L})$ of D-lactate by fed-batch fermentation. This titer is slight lower than the $113.1 \mathrm{~g} / \mathrm{L}$ of $\mathrm{D}$-lactate which was obtained 


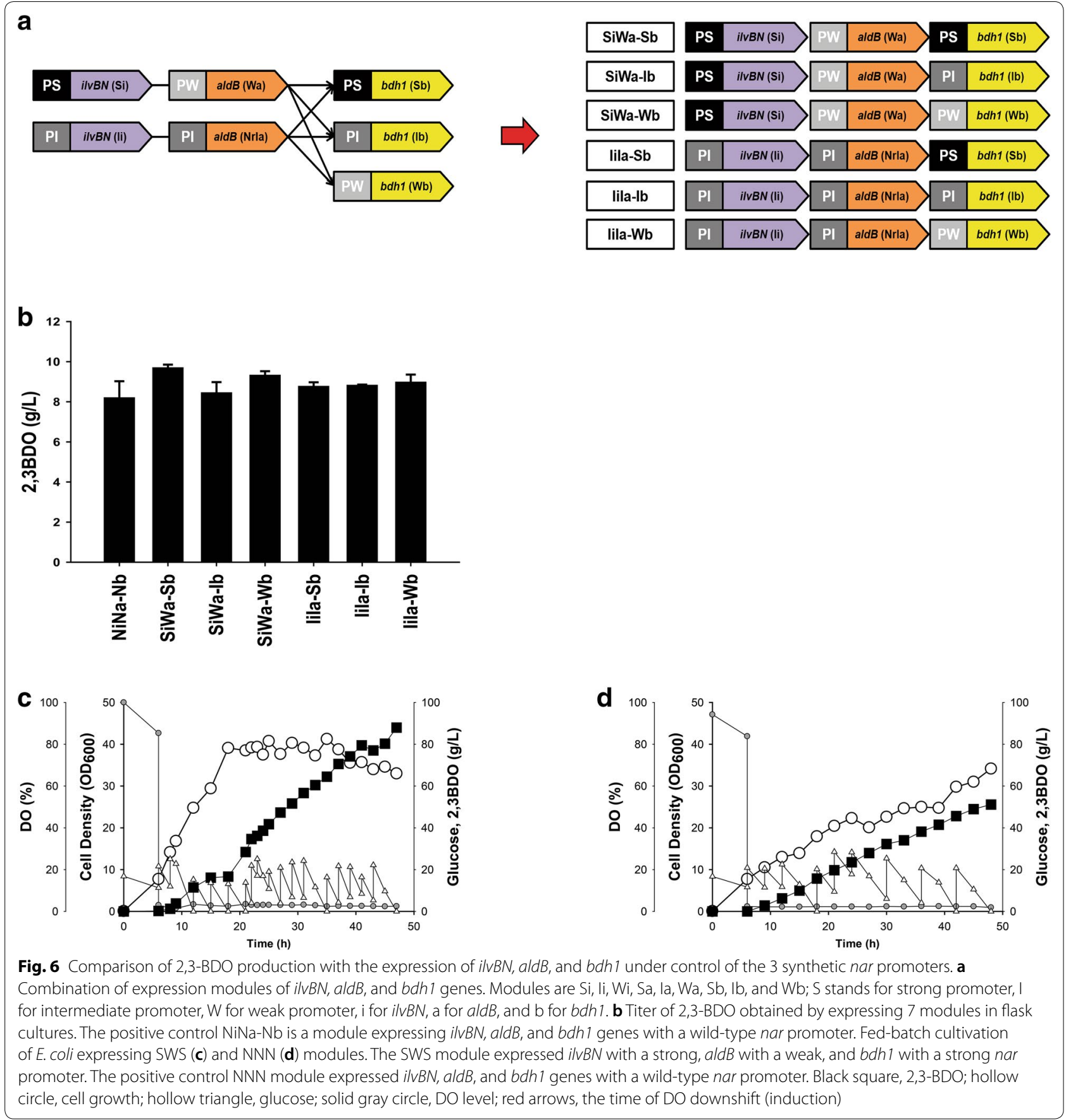

by fed-batch fermentation [13] by expression of the $l d h D$ gene with the wild-type nar promoter on a highcopy plasmid. This suggests that the $l d h D$ expression level may be highly correlated with titer of D-lactate. The correlation of expression level and production titer was also high in the production of 2,3-BDO. In a similar manner to D-lactate pathway gene expression, each of the three 2,3-BDO biosynthesis pathway genes $(i l v B N, a l d B$, and $b d h 1)$ were modified to be under the control of the synthetic nar promoters of different strengths and combinatorially expressed. In the case of the acetoin pathway, which is a precursor of 2,3-BDO, controlling ilvBN gene expression was critical in biosynthesis flux because the expression of $i l v B N$ with a weak nar promoter resulted in lower titers of acetoin than expression with strong or intermediate promoters regardless of the promoter strength for expressing the ald $B$ gene. The acetoin pathway was further extended 
to 2,3-BDO by controlling expression of the $b d h 1$ gene with the 3 synthetic nar promoters. The best combinatorial expression module for 2,3-BDO was a strong promoter for $i l v B N(\mathrm{Si})$, weak promoter for $\operatorname{ald} B(\mathrm{Wa})$, and strong promoter for $b d h 1(\mathrm{Sb})$. The expression module $(\mathrm{Si}+\mathrm{Wa}+\mathrm{Sb})$ produced $88.0 \mathrm{~g} / \mathrm{L}$ of $2,3-\mathrm{BDO}$ in fedbatch fermentation, which was $72 \%$ higher than the $51.1 \mathrm{~g} / \mathrm{L}$ obtained by expressing the 3 enzymes with a wild-type nar promoter.

\section{Conclusions}

In this study, the synthetic nar promoters, which were engineered to have strong, intermediate, and weak intensities, were successfully applied to metabolic engineering of the D-lactate and 2,3-BDO pathways in E. coli. By controlling expression levels of one D-lactate enzyme and three 2,3-BDO pathway enzymes using the synthetic nar promoters, the production of D-lactate and 2,3-BDO was increased by 34 and $72 \%$, respectively, compared with production using a wild-type nar promoter. This synthetic nar promoter module system will support the improved production of biochemicals and biofuels through the fine-tuning of gene expression levels in $E$. coli.

\section{Methods}

\section{Strains}

The E. coli TOP10 (Invitrogen, USA) strain was used for cloning and maintenance of plasmids. The E. coli W023 $[13,21]$ strain was used to produce D-lactate, acetoin, and 2,3-BDO in flask and fed-batch fermentation. The bacterial strains used in this study are listed in Table 1.

\section{Construction of the randomized nar promoter library}

A gfpm gene encoding GFPm was amplified by PCR from $\mathrm{pQE}-$ gfpm [22] with gene-specific primers including ribosome binding site (RBS) and restriction enzyme sites, and then the PCR product was inserted between $X m a \mathrm{I}$ and NotI sites downstream of a wild-type nar promoter on pUCN [13] and pUCM plasmids [23]. In order to randomize the spacer sequence $(15 \mathrm{bp})$ between the -10 and -35 elements of the nar promoter, two primers, XmaI-SNPL-gfpm-F and SphI-gfpm-R (Table 2) were designed. The XmaI-SNPL-gfpm-F primer contains a restriction enzyme site (XmaI), the -35 element, randomized 15-bp sequences $\left(\mathrm{N}_{15}\right)$, the -10 element, and the gfpm-specific sequence in order. The SphI-gfpm-R primer contains a restriction enzyme site $(S p h \mathrm{I})$ and the gfpm-specific sequence. The randomized nar promoter region and $g f p m$ gene amplified by PCR were cloned into the XmaI and SphI sites of the pSTVM2 plasmid [13], generating pSTVM2-SNPL-gfpm. The pSTVM2-SNPLgfpm plasmids were transduced into $E$. coli TOP10 cells by electroporation and the transformants were incubated in $40 \mathrm{~mL}$ Luria-Bertani (LB) medium supplemented with $30 \mu \mathrm{g} / \mathrm{L}$ chloramphenicol [24] at $30{ }^{\circ} \mathrm{C}$ with shaking at $100 \mathrm{rpm}$.

\section{Screening of the randomized nar promoter library}

Escherichia coli cells harboring pSTVM2-SNPL-gfpm were cultivated in $40 \mathrm{~mL} \mathrm{LB}$ medium supplemented with $50 \mu \mathrm{g} / \mathrm{mL} \mathrm{Cm}$ in a $100-\mathrm{mL}$ flask at $30^{\circ} \mathrm{C}$ with shaking at $100 \mathrm{rpm}$ for $12 \mathrm{~h}$. Cells were harvested by centrifugation at $7000 \mathrm{rpm}$ for $10 \mathrm{~min}$ at $4{ }^{\circ} \mathrm{C}$ and washed twice with phosphate buffered saline (PBS, $137 \mathrm{mM} \mathrm{NaCl}, 2.7 \mathrm{mM}$ $\mathrm{KCl}, 10 \mathrm{mM} \mathrm{Na} \mathrm{HPO}_{4}$, and $2 \mathrm{mM} \mathrm{KH} \mathrm{PO}_{4}, \mathrm{pH}$ 7.4). After washed cells were resuspended with PBS buffer, cells were subjected to a fluorescent activated cell sorter (FACS; MoFlo XDP, Beckman Coulter, FL). FACS-sorted cells were directly poured into fresh LB agar plates containing $\mathrm{Cm}(50 \mu \mathrm{g} / \mathrm{mL})$ and incubated at $30{ }^{\circ} \mathrm{C}$ for $12 \mathrm{~h}$. Cells scraped from the plates were then cultivated in $40 \mathrm{~mL}$ LB medium containing $\mathrm{Cm}(50 \mu \mathrm{g} / \mathrm{mL})$ in a 100 $\mathrm{mL}$ flask and then subjected to the next round of FACS sorting following the same procedure mentioned above. After the third round of FACS sorting, colonies on LB agar plates were randomly selected to cultivate in $200 \mu \mathrm{L}$ of $\mathrm{LB}+\mathrm{Cm}$ medium in a 96-deep-well plate at $30^{\circ} \mathrm{C}$ with shaking at $100 \mathrm{rpm}$ overnight.

\section{Construction of plasmids}

The nucleotide sequences of the primers used in construction of plasmids are listed in Table 2. Plasmid pUCN [13] was modified by adding the rop gene, which resulted in a low-copy number plasmid pUCNr with a wild-type nar promoter. Next, the wild-type nar promoter in pUCNr was replaced by one of the three representative synthetic nar promoters [S3-2-64 (strong), W2U-30 (moderate), W2L-29 (weak)] and amplified by PCR with each synthetic promoter-specific reverse primer and phosphorylated -10Pnar-F primer. The constructed plasmids were named pUCNrS (for S3-2-64), pUCNrI (for W2U-30), and pUCNrW (for W2L-29, weak promoter). The reporter $g f p m$ gene was amplified by PCR from pQE-gfpm [22] with primers (XmaI-gfpm$\mathrm{F}$ and NotI-gfpm-R) and cloned downstream of the lac promoter of pUCM, generating pUCM-gfpm. To obtain pSTVM-gfpm, the PCR-amplified gfpm gene containing the lac promoter was inserted between the BamHI and EcoRI sites of the pSTVM2 plasmid [12]. The $l d h D$ encoding D-lactate dehydrogenase of Leuconostoc citreum, ilvBN-encoding acetohydroxy acid synthase of $E$. coli, aldB-encoding acetolactate decarboxylase of Lactococcus lactis, and bdh1-encoding butanediol dehydrogenase from Saccharomyces cerevisiae were amplified by PCR from the genomic DNAs of each strain, and then 
Table 2 Primers used in this study

\begin{tabular}{|c|c|}
\hline Primer name & Sequence $\left(5^{\prime}-3^{\prime}\right)$ \\
\hline \multicolumn{2}{|l|}{ For cloning } \\
\hline Xmal-SNPL-gfpm-F & $\begin{array}{l}\text { TCCCCCCCGGGCTCTTGATCGTTATCAAATCCCANNNNNNNNNNNNNNNNGTATAA } \\
\text { TGCCCTTAAAAGGAGGATTACAAAATGAGTAAAGGAGAAGAACT }\end{array}$ \\
\hline Sphl-gfpm-R & ACATGCATGCTTAGTGGTGGTGGTGGTGGTGTTTGTAGAGCTCATCGATGC \\
\hline Xmal-gfpm-F & TCCCCCCGGGAGGAGGATTACAAAATGAGTAAAGGAGAAGAACTTTT \\
\hline Notl-gfpm-R & TAAGAATGCGGCCGCTTAGTGGTGGTGGTGGTGGTGTTTGTAGAGCTCATCGATGC \\
\hline IdhD-citreum-F-Xbal & CTAGTCTAGAAGGAGGATTACAAAATGAAGATTTTTGCTTATGGT \\
\hline IdhD-citreum-R-Notl & TTCCCTT GCGGCCGCTTAATACTTTACAGCAATACTT \\
\hline ilvB-EC-F-Xbal & CTAGTCTAGAAGGAGGATTACAAAATGGCAAGTTCGGGCA \\
\hline ilvN-EC-R-Notl & TTCCCTTGCGGCCGCTTACTGAAAAAACACCGCGAT \\
\hline aldB-LL-F-Xbal & CTAGTCTAGAAGGAGGATTACAAAATGACAGAAATCACACAACTT \\
\hline aldB-LL-R-Notl & TTCCCTTGCGGCCGCTCATTCAGCTACATCGATATC \\
\hline bdh1-SC-F-Xmal & TCCCCCCGGGAGGAGGATTACAAAATGAGAGCTTTGGCATATTTC \\
\hline bdh1-SC-R-Notl & TTCCCTTGCGGCCGCTTACTTCATTTCACCGTGATT \\
\hline Sphl-pUC-F & ACAT GCATGCCCGACTGGAAAGCG \\
\hline Sphl-pUC-R & ACATGCATGCCGGTGTGAAATACCG \\
\hline Pstl-pUC-F & AAAACTGCAGCCGACTGGAAAGCG \\
\hline BamHI-pUC-R & CGGGATCCCGGTGTGAAATACCG \\
\hline PnarS-R & TCTCTAACACAGTATTGGGATTTGATAACGATCAAG \\
\hline Pnarl-R & AATCTAGTAACCCGATGGGATTTGATAACGATCAAG \\
\hline PnarW-R & GGAGATGTTACAATATGGGATTTGATAACGATCAAG \\
\hline-10 Pnar-F & GTATAATGCCCTTAAATCTAGA \\
\hline pUCN-ori-fr & AGGAAGCGGAAGAGCG \\
\hline pUCN-ori-r & GAAGATCCTTTGATCTTTTCTA \\
\hline pET-ori-R & TTGAGATCCTTTTTTTCTGC \\
\hline pET-rop-F & GGTGCGCATGATCGTG \\
\hline pSTVM2-pUC-sub-USER-3-F & AGACAGUCATAAGTGCGG \\
\hline pSTVM2-pUC-sub-USER-1-R & ATGCAACUCGTAGGACAG \\
\hline pUC-sub-USER-1-F & AGTTGCAUCCCGACTGGAAAGCG \\
\hline pUC-sub-USER-2-F & ATCCATGUCCCGACTGGAAAGCG \\
\hline pUC-sub-USER-5-F & ATATGCGAUCCCGACTGGAAAGCG \\
\hline pUC-sub-USER-2-R & ACATGGAUATGCGGTGTGAAATACC \\
\hline PUC-sub-USER-5-R & ATCGCATAUATGCGGTGTGAAATACCG \\
\hline pUC-sub-USER-3-R & ACTGTCUATGCGGTGTGAAATACCG \\
\hline \multicolumn{2}{|l|}{ For qRT-PCR } \\
\hline q-cysG-F & TTGTCGGCGGTGGTGATGTC \\
\hline q-cysG-R & ATGCGGTGAACTGTGGAATAAACG \\
\hline q-gfpm-F & AGAGGGTGAAGGTGATGCCA \\
\hline q-gfpm-R & AGATGATCCGGATAACGCGC \\
\hline
\end{tabular}

Italic and underline letters represent a restriction enzyme site

cloned downstream of the synthetic nar promoters of pUCNrS, pUCNrI, and pUCNrW (Table 2). For complementation experiments, the $i l v B N$ gene was amplified by PCR with the synthetic nar promoter and a terminator, and then inserted between the PstI and BamHI sites of the pSTVM2 plasmid. To assemble two genes ( $i l v B N$ and aldB) encoding acetoin pathway enzymes and three genes (ilvBN, aldB, and bdh1) encoding 2,3-BDO pathway enzymes in pSTVM2, each gene was amplified by PCR with the synthetic nar promoter and a terminator, and then subcloned into PSTVM2 using the USER ${ }^{\mathrm{TM}}$ cloning method [25, 26].

\section{Flask and bioreactor fermentations}

The recombinant $E$. coli strains harboring a plasmid or plasmids for production of D-lactate, acetoin, and 
2,3-BDO were inoculated in $4 \mathrm{~mL} \mathrm{LB}$ medium supplemented with $50 \mu \mathrm{g} / \mathrm{mL} \mathrm{Cm}$ or/and $100 \mu \mathrm{g} / \mathrm{mL}$ ampicillin (Ap) at $37^{\circ} \mathrm{C}$ overnight with shaking at $250 \mathrm{rpm}$. For flask cultivation, 100 -mL flasks were filled with $40 \mathrm{~mL} \mathrm{LB}$ medium containing $20 \mathrm{~g} / \mathrm{L}$ glucose and appropriate antibiotics, and then were inoculated with $2 \%(\mathrm{v} / \mathrm{v})$ seed culture. For D-lactate production, $\mathrm{pH}$ of the culture media was controlled by adding $10 \mathrm{~g} / \mathrm{L} \mathrm{CaCO}_{3}$. A nar promoter was induced by reducing shaking speed from 250 to $100 \mathrm{rpm}$ when recombinant $E$. coli cells grew to an $\mathrm{OD}_{600}$ of 1.0 at $30{ }^{\circ} \mathrm{C}$ at $250 \mathrm{rpm}$. For bioreactor fermentation, fed-batch culturing was carried out with an initial culture volume of $1.0 \mathrm{~L}$ of modified R [27] medium containing $20 \mathrm{~g} / \mathrm{L}$ glucose, $5 \mathrm{~g} / \mathrm{L}$ yeast extract, and the required antibiotics in a 3.0-L jar bioreactor BIOSTAT B (Sartorius, Germany) [28]. The temperature was maintained at $30{ }^{\circ} \mathrm{C}$ and $\mathrm{pH}$ was automatically controlled at 7.0 by adding $5 \mathrm{~N}$ $\mathrm{NH}_{4} \mathrm{OH}$. The DO level was controlled by supplying air or a mixture of air and pure oxygen gas. In order to induce nar promoters, cells were grown at DO level $>80 \%$ (aerobic phase) until an $\mathrm{OD}_{600}$ of 10.0 and then immediately DO level was decreased to $<1-2 \%$ (microaerobic). The feeding solution consisting of $800 \mathrm{~g} / \mathrm{L}$ glucose, $50 \mathrm{~g} / \mathrm{L}$ yeast extract, $15 \mathrm{~g} / \mathrm{L}$ tryptone, $15 \mathrm{~g} / \mathrm{L} \mathrm{MgSO}_{4} \cdot 7 \mathrm{H}_{2} \mathrm{O}$, and $5 \mathrm{~g} / \mathrm{L}_{\mathrm{KH}_{2}} \mathrm{PO}_{4}$ [29] was periodically added when the residual glucose concentration was below $5-10 \mathrm{~g} / \mathrm{L}$. Cell growth was monitored at a wavelength of $600 \mathrm{~nm}$ with a SPECTRAmax PLUS384 (Molecular Devices, USA).

\section{Transcriptional analysis}

Cells were grown in LB medium containing 2\% (w/v) glucose until mid-exponential growth phase, and total RNA was extracted using Hybrid-R RNA purification kit (GeneAll biotechnology, Korea) according to the manufacturer's instructions. Quantitative reverse transcription PCR (qRT-PCR) was performed using a Rotor-Gene Q (Qiagen, Germany) and SensiFAST ${ }^{\mathrm{TM}}$ SYBR No-ROX One-Step Kit (Bioline, USA). Solutions of $5.0 \mu \mathrm{L}$ of $2 \times$ SensiFAST $^{\mathrm{TM}}$ SYBR No-ROX One-Step mix, $0.1 \mu \mathrm{L}$ of reverse transcriptase, $0.2 \mu \mathrm{L}$ of RNase inhibitor, $0.4 \mathrm{mM}$ forward and reverse primers (gfpm-qPCR-F and gfpm-qPCR-R), $2.0 \mu \mathrm{L}$ of isolated total RNA (10 ng/ $\mu \mathrm{L}$ ), and $1.9 \mu \mathrm{L}$ of diethylpyrocarbonate (DEPC)-treated water were mixed for each qRT-PCR reaction and qRTPCR was performed as follow: $45^{\circ} \mathrm{C}$ for $10 \mathrm{~min}, 95{ }^{\circ} \mathrm{C}$ for $2 \mathrm{~min}$, and then 40 cycles of $95^{\circ} \mathrm{C}$ for $5 \mathrm{~s}, 60^{\circ} \mathrm{C}$ for $10 \mathrm{~s}$, and $72{ }^{\circ} \mathrm{C}$ for $5 \mathrm{~s}$. The value of $\Delta \Delta C_{\mathrm{t}}$ was averaged from triplicate measurements. The cys $G$ gene encoding siroheme synthase was used as a reference gene and the genes expressed by the wild-type nar promoter were used as calibrators.

\section{Western blotting analysis}

GFPm expression under the control of nar promoters was analyzed by western blotting. The harvest cells were washed and resuspended in $20 \mathrm{mM}$ Tris- $\mathrm{HCl}(\mathrm{pH}$ 8.0), and then disrupted by sonication. After centrifugation, supernatants were collected, quantified using the Bradford method, and then analyzed by $15 \%$ (w/v) SDSPAGE. For immunodetection of His-tagged GFPm, a monoclonal anti-polyhistidine antibody (Sigma-Aldrich, USA) and horseradish peroxidase-conjugated anti-mouse IgG (Pierce, USA) were used according to the manufacturer's instructions. GAPDH was used as a reference gene for quantification of proteins.

\section{Fluorescence analysis}

After cells were grown under aerobic conditions, GFPm protein expression under the control of nar promoters was induced by lowering DO levels through changing culture rpm of 250-100. Harvested cells were washed and resuspended in $1 \mathrm{~mL}$ phosphate buffered saline (PBS, $137 \mathrm{mM} \mathrm{NaCl}, 2.7 \mathrm{mM} \mathrm{KCl}, 10 \mathrm{mM} \mathrm{Na}_{2} \mathrm{HPO}_{4}$, and $2 \mathrm{mM} \mathrm{KH}_{2} \mathrm{PO}_{4}, \mathrm{pH}$ 7.4) and the fluorescence intensity of the reporter GFPm protein was measured using a SPECTRAmax Gemini plate reader (Molecular Devices, USA) with excitation at $470 \mathrm{~nm}$ and emission at $510 \mathrm{~nm}$. Cytometric analysis was performed using a BD FACS Calibur flow cytometer (BD Biosciences, USA). GFPm was excited using a $15-\mathrm{mW}$ argon ion laser $(488 \mathrm{~nm})$ and fluorescence emission was detected using the FL1 channel (530/30 bandpass filter).

\section{Metabolite analysis}

The concentrations of glucose, D-lactate, 2,3-BDO, and other metabolites were determined using an Agilent Technologies 1200 high-performance liquid chromatography equipped with a refractive index detector (Agilent, USA) and an Aminex HPX-87H column (Bio-Rad, USA) at a flow rate of $0.7 \mathrm{~mL} / \mathrm{min}$ and column temperature of $50{ }^{\circ} \mathrm{C}$ using $4 \mathrm{mM} \mathrm{H}_{2} \mathrm{SO}_{4}$ as the mobile phase.

\section{Abbreviations \\ GFP: green fluorescent protein; 2,3-BDO: 2,3-butanediol; FACS: fluorescent activated cell sorter; RFU: relative fluorescence unit.}

\section{Authors' contributions}

$\mathrm{HJH}$ participated in the experimental design and work, data analysis, and writing of the manuscript. SYL assisted in the experimental design. PCL assisted in the experimental design, the experimental work, data analysis, and writing of the manuscript. All authors read and approved the final manuscript.

\section{Author details}

${ }^{1}$ Department of Molecular Science and Technology, Ajou University, Woncheon-dong, Yeongtong-gu, Suwon 16944, South Korea. ${ }^{2}$ Department of Chemical and Biomolecular Engineering, KAIST, Daejeon 34141, South Korea. 


\section{Acknowledgements \\ Not applicable.}

\section{Competing interests}

The authors declare that they have no competing interests.

\section{Availability of data and materials \\ Not applicable.}

\section{Consent for publication}

Not applicable.

\section{Ethics approval and consent to participate}

Not applicable.

\section{Funding}

This work was supported by a National Research Foundation of Korea Grant funded by the South Korean Government (2012M1A2A2671691, 2009-0093826).

\section{Publisher's Note}

Springer Nature remains neutral with regard to jurisdictional claims in published maps and institutional affiliations.

\section{Received: 1 August 2017 Accepted: 2 April 2018}

Published online: 07 April 2018

\section{References}

1. Boyle PM, Silver PA. Parts plus pipes: synthetic biology approaches to metabolic engineering. Metab Eng. 2012;14(3):223-32.

2. Connor MR, Atsumi S. Synthetic biology guides biofuel production. J Biomed Biotechnol. 2010;2010. https://doi.org/10.1155/2010/541698.

3. Guiziou S, Sauveplane V, Chang HJ, Clerte C, Declerck N, Jules M, Bonnet J. A part toolbox to tune genetic expression in Bacillus subtilis. Nucleic Acids Res. 2016:44(15):7495-508.

4. Shive E, Prather KLJ. Synthetic biology devices as tools for metabolic engineering. Biochem Eng J. 2012;65:82-9.

5. Alper H, Fischer C, Nevoigt E, Stephanopoulos G. Tuning genetic control through promoter engineering. Proc Natl Acad Sci USA 2006;103:12678-83

6. Nevoigt E, Kohnke J, Fischer CR, Alper H, Stahl U, Stephanopoulos G Engineering of promoter replacement cassettes for fine-tuning of gene expression in Saccharomyces cerevisiae. Appl Environ Microbiol. 2006;72(8):5266-73

7. Blazeck J, Alper HS. Promoter engineering: recent advances in controlling transcription at the most fundamental level. Biotechnol J. 2013;8(1):46-58.

8. Hammer K, Mijakovic I, Jensen PR. Synthetic promoter libraries-tuning of gene expression. Trends Biotechnol. 2006;24(2):53-5.

9. Watanabe D, Kaneko A, Sugimoto Y, Ohnuki S, Takagi H, Ohya Y. Promoter engineering of the Saccharomyces cerevisiae RIM15 gene for improvement of alcoholic fermentation rates under stress conditions. J Biosci Bioeng. 2017:123(2):183-9.

10. Gilman J, Love J. Synthetic promoter design for new microbial chassis Biochem Soc Trans. 2016:44(3):731-7.

11. Mahr R, von Boeselager RF, Wiechert J, Frunzke J. Screening of an Escherichia coli promoter library for a phenylalanine biosensor. Appl Microbiol Biotechnol. 2016;100(15):6739-53.

12. Schofield DM, Templar A, Newton J, Nesbeth DN. Promoter engineering to optimize recombinant periplasmic Fab' fragment production in Escherichia coli. Biotechnol Prog. 2016;32(4):840-7.

13. Hwang HJ, Kim JW, Ju SY, Park JH, Lee PC. Application of an oxygeninducible nar promoter system in metabolic engineering for production of biochemicals in Escherichia coli. Biotechnol Bioeng. 2017;114(2):468-73.

14. Pröschel M, Detsch R, Boccaccini AR, Sonnewald U. Engineering of metabolic pathways by artificial enzyme channels. Front Bioeng Biotechnol. 2015:3:168
15. Castellana M, Wilson MZ, Xu Y, Joshi P, Cristea IM, Rabinowitz JD, Gitai Z, Wingreen NS. Enzyme clustering accelerates processing of intermediates through metabolic channeling. Nat Biotechnol. 2014;32(10):1011-8.

16. Chubukov V, Mukhopadhyay A, Petzold CJ, Keasling JD, Martín HG. Synthetic and systems biology for microbial production of commodity chemicals. NPJ Syst Biol Appl. 2016;2:16009.

17. Zhou L, Zuo ZR, Chen XZ, Niu DD, Tian KM, Prior BA, Shen W, Shi GY, Singh S, Wang ZX. Evaluation of genetic manipulation strategies on D-lactate production by Escherichia coli. Curr Microbiol. 2011;62(3):981-9.

18. Liu M, Tolstorukov M, Zhurkin V, Garges S, Adhya S. A mutant spacer sequence between -35 and - 10 elements makes the Plac promoter hyperactive and CAMP receptor protein-independent. Proc Natl Acad Sci USA. 2004;101(18):6911-6.

19. Nielsen DR, Yoon SH, Yuan CJ, Prather KL. Metabolic engineering of acetoin and meso-2,3-butanediol biosynthesis in E. coli. Biotechnol J. 2010;5(3):274-84

20. Hook-Barnard IG, Hinton DM. The promoter spacer influences transcription initiation via $\sigma 70$ region 1.1 of Escherichia coli RNA polymerase. Proc Natl Acad Sci USA. 2009;106(3):737-42.

21. Hwang HJ, Park JH, Kim JH, Kong MK, Kim JW, Park JW, Cho KM, Lee PC. Engineering of a butyraldehyde dehydrogenase of Clostridium saccharoperbutylacetonicum to fit an engineered 1,4-butanediol pathway in Escherichia coli. Biotechnol Bioeng. 2014;111(7):1374-84.

22. Yoo TH, Link AJ, Tirrell DA. Evolution of a fluorinated green fluorescent protein. Proc Natl Acad Sci USA. 2007;104(35):13887-90.

23. Kim SH, Park YH, Schmidt-Dannert C, Lee PC. Redesign, reconstruction, and directed extension of the Brevibacterium linens C40 carotenoid pathway in Escherichia coli. Appl Environ Microbiol. 2010;76(15):5199-206.

24. Brown JL, Ross T, McMeekin TA, Nichols PD. Acid habituation of Escherichia coli and the potential role of cyclopropane fatty acids in low pH tolerance. Int J Food Microbiol. 1997:37(2-3):163-73.

25. Cavaleiro AM, Kim SH, Seppala S, Nielsen MT, Norholm MH. Accurate DNA assembly and genome engineering with optimized uracil excision cloning. ACS Synth Biol. 2015;4(9):1042-6.

26. Nørholm MH. A mutant Pfu DNA polymerase designed for advanced uracil-excision DNA engineering. BMC Biotechnol. 2010;10(1):1-7.

27. Gonzalez MD, Lichtensteiger CA, Caughlan R, Vimr ER. Conserved filamentous prophage in Escherichia coli O18:K1:H7 and Yersinia pestis biovar orientalis. J Bacteriol. 2002;184:6050-5.

28. Jung YK, Kim TY, Park SJ, Lee SY. Metabolic engineering of Escherichia coli for the production of polylactic acid and its copolymers. Biotechnol Bioeng. 2010:105(1):161-71.

29. Han SJ, Chang HN, Lee J. Characterization of an oxygen-dependent inducible promoter, the nar promoter of Escherichia coli, to utilize in metabolic engineering. Biotechnol Bioeng. 2001;72(5):573-6.
Ready to submit your research? Choose BMC and benefit from:

- fast, convenient online submission

- thorough peer review by experienced researchers in your field

- rapid publication on acceptance

- support for research data, including large and complex data types

- gold Open Access which fosters wider collaboration and increased citations

- maximum visibility for your research: over 100M website views per year

At BMC, research is always in progress.

Learn more biomedcentral.com/submissions 